\title{
ADHD theories still need to take more on board: Serotonin and pre-executive variability
}

\author{
Robert D. Oades and Hanna Christiansen
}

Behavioral and Brain Sciences (2005), 28, 438

DOI:10.1017/S0140525X0533007X

Biopsychology Group, University Clinic for Child and Adolescent Psychiatry, 45147 Essen, Germany. Email: robert.oades@uni-due.de

Commentary on

Terje Sagvolden, Espen Borga Johansen, Heidi Aase, and Vivienne Ann Russell.

"A dynamic developmental theory of Attention-Deficit/Hyperactivity Disorder (ADHD) predominantly hyperactive/impulsive and combined subtypes"

Behavioral and Brain Sciences (2005), 28, 397-468 (Abstract at the end)

\begin{abstract}
:
Correcting the relationship between tonic and burst firing modes in dopamine neurons may help normalize stimulus-reinforcement gradients and contingent behavior in ADHD children. But appropriate evaluations of stimuli for developing adaptive plans and controlling impulsivity will not occur without moderating the gain-like functions of serotonin. The "Dynamic Theory" correctly highlights the need to account for variability in ADHD. The dysmaturation of pre-executive information processing is proposed as an explanation.

At the core of the article by Sagvolden and colleagues there is a set of data that throws light on an aspect of the ADHD phenomenon. But one asks if the authors are a measure too brave to generalize so broadly from the unusually steep reinforcement gradients reported for the human condition and an animal model to the syndrome as a whole.
\end{abstract}

\section{Commentary:}

Sagvolden et al acknowledge that transmitter systems other than the dopaminergic pathways are likely to be involved in causing or mediating the features of the ADHD condition. So it would be unfair to emphasize the potential pathophysiological contributions of these transmitters too much. The problem is that they impinge on the core of the hypothesis proposed.

For example, the "Dynamic Theory" does not take account of a role for serotonin $(5-\mathrm{HT})$. One notes that, several agents (e.g. amphetamine, cocaine) act presynaptically and affect dopamine (DA) transport. Amphetamine has a therapeutic effect. But both alter 5-HT dynamics. Indeed if the DA transporter is knocked out in rodents reinforcement measured by cocaine administration (Mateo et al. 2004) or conditioned place preference to amphetamine (Budygin et al. 2004) remains, until a $5-\mathrm{HT}_{1 \mathrm{a}}$ antagonist is administered. In ADHD children cognitive impulsivity measured by a reduced probability of inhibition in the stop-task, is associated with decreased affinity (increased $\mathrm{Kd}$ in platelets) of the $5-\mathrm{HT}$ transporter (Oades et al. 2002). In continuous performance tests, perceptual sensitivity falls with an increased excretion of 5-HT metabolites (Oades 2000). The relationship of DA to 5-HT activity (HVA/5HIAA) seems depressed in some samples of ADHD children (Oades 2002), although increases of this ratio may reflect motor activity (Castellanos et al. 1994). Thus there is reason to believe that $5-\mathrm{HT}$ plays a 
marked role in the sensory, reinforcement, inhibitory and motor processes that are disturbed in ADHD.

Our argument would imply, at least in relation to 5-HT activity that the DA system is hypoactive. We seem to be partially in agreement with Sagvolden et al. on DA "hypo-activity". Certainly, the stimulant nature of methylphenidate that acts at catecholaminergic and not 5-HT sites seems to be consistent with this standpoint. However, if this is so one must find an explanation for how ADHD phenomenology can co-exist with Tic/Tourette syndromes, where psychostimulants are contra-indicated and neuroleptics can ameliorate. Can ADHD symptoms co-exist with what appears to be a hyperactive DA system?

Sagvolden et al refer to the potentially crucial difference between the tonic and burst firing modes of ascending DA neurons elaborated by Grace (2001: pp.26-27). Herein could lie the answer to the Tic/ADHD conundrum. Putatively, the tonic level of DA neuron firing is high in Tic-patients and lies close to the threshold for eliciting burst firing. Psychostimulants may then raise spontaneous firing levels such that the threshold for burst firing is exceeded more often, analogous to the elicitation of sterotypies in rodents with high doses of amphetamine. For ADHD patients, starting from lower levels of tonic firing (hypoactivity), this upgrading of sensitivity to DA may be of course "just what the doctor ordered". However this still begs the question whether the core of ADHD problems (that can also be found in Tic-patients) lies outside the direct influence of DA. Perhaps the mere promotion of the likelihood of DA function in and around the synapse is helpful, but indirectly so.

Let us return to the 'core of ADHD problems'. Sagvolden et al (also Castellanos \& Tannock 2002) highlight the variability of response as one of the central features of ADHD. A prediction of the "Dynamic Theory" is that this variability arises from the "extra-nominal" class or unusual response pattern (for a given situation) becoming 'the rule'. This is an interesting and unusual form of 'persistence', but as such is consistent with hypo-dopaminergic function. The function that we note here is the role of increasing DA activity in initiating action or promoting the likelihood of a switch between competing actions, as proposed and demonstrated elsewhere (Oades 1985; Oades 1997). Sagvolden et al. also expressly note that the function of burstfiring DA neurons lies in the initiation of behavior (p.15). Thus to a degree we agree on the so-called role of ascending DA activity. But we also note it is consistent with the more parsimonious idea of DA having a general role in competitive information processing rather than specific reinforcement (Oades, 1999).

Important for the discussion here is that there is an alternative explanation for the variability of behavior in ADHD. Namely, that there is an impairment in top-down control of processing incoming information. This control may be independent of DA, although it may be markedly influenced by an impaired role of noradrenaline in 'tuning' different inputs. This viewpoint may account for a range of anomalous features of ADHD not incorporated by the impairment of perception and integration of reinforcement described in the 'Dynamic Theory'.

There are examples from the control of attention. Steady state visual potential latencies in ADHD suggest a decreased efficiency in coupling between PRF networks, especially in the right hemisphere (Silberstein et al. 1998). Reduced speeds of conduction (Ucles et al. 1996) and delayed latencies (e.g. P1, N1: Karayanidis et al. 2000; Johnstone et al. 
2001) in late developing regions (e.g. delayed myelination) would form a good basis for response variability and poor time perception (Rubia et al. 2003; Toplak et al. 2003). The delay could also account for the slowed orienting of attention by the right hemisphere to the left visual field and evaluation of the cue eliciting the orientation (McDonald et al. 1999, Oie et al. 1998; Carter et al. 1995). With such "inefficient coupling" it is no surprise that event-related recordings show poor differentiation of Go, No-go stimuli and errors in the stages of information processing that follow (Dimoska et al. 2003; Liotti et al. 2004). Further this hypothesis is consistent with data supporting an etiology in terms of a maturational lag (Cantwell 1985), with the delay particularly affecting those parts of the frontal lobe that develop last.

Part of our suggestion may be consistent with part of the 'Dual Pathway' hypothesis (Sonuga-Barke 2003). Undoubtedly the "Dynamic Theory" with its emphasis on mesolimbic reinforcement mechanisms finds support from another part of the dual pathway. Sagvolden and colleagues make a major constructive contribution to the continuing need to try to account for all the data.

\section{References:}

Budygin, E. A., Brodie, M. S., Sotnikova, T. D., Mateo, Y., John, C. E., Cyr, M. et al. (2004) Dissociation of rewarding and dopamine transporter-mediated properties of amphetamine. Proceedings of the National Academy of Sciences (U.S.A), 101: 7781-7786.

Cantwell, D. P. (1985) Hyperactive children have grown up: what have we learned about what happens to them? Archives of General Psychiatry, 42: 1026-1028.

Carter, C. S., Krener, P., Chaderjian, M. C., Northcutt, C. \& Wolfe, V. (1995) Asymmetrical visual-spatial attentional performance in ADHD: evidence for a right hemisphere deficit. Biological Psychiatry, 37: 789-707.

Castellanos, F. X., Elia, J., Kruesi, M. J. P., Gulotta, C. S., Mefford, I. N., Potter, W. Z. et al. (1994) Cerebrospinal fluid monoamine metabolites in boys with attention-deficit hyperactivity disorder. Psychiatry Research, 52: 305-316.

Castellanos, F. X., \& Tannock, R. (2002) Neuroscience of attentiondeficit/hyperactivity disorder: the search for endophenotypes. Nature Reviews: Neuroscience, 3: 617-628.

Dimoska, A. Johnstone, S. J., Barry, R. J., \& Clarke, A. R. (2003) Inhibitory motor control in children with attentiondeficit/hyperactivity disorder: eventrelated potentials in the stop-signal paradigm. Biological Psychiatry, 54: 1345-1354.

Grace, A. A. (2001) Psychostimulant actions on dopamine and limbic system function: Relevance to the pathophysiology and treatment of ADHD. In: Stimulant drugs and ADHD: Basic and clinical neuroscience: pp. 134-157, M. V. Solanto, A. F. T. Arnsten, \& F. X. Castellanos (Eds.), Oxford University Press.

Johnstone, S. J. Barry, R. J. \& Anderson, J. W. (2001) Topographic distribution and developmental time course of auditory event-related potentials in two subtypes of attention-deficit hyperactivity disorder. International Journal of Psychophysiology, 42: 73-94

Karayanidis, F., Robaey, P., Bourassa, M., de Koning, D., Geoffroy, G., \& Pelletier, G. (2000) ERP differences in visual attention processing between attention-deficit hyperactivity disorder and control boys in the absence of performance differences. Psychophysiology, 37: 319-333.

Liotti, M., Pliszka, S. R., Perez, R., Kothmann, F. D., \& Woldorff, M. G. (2004) Abnormal brain activity related to performance monitoring and error 
detection in children with ADHD. Cortex, in press.

Mateo, Y., Budygin, E. A., John, C. E., \& Jones, S. R. (2004) Role of serotonin in cocaine effects in mice with reduced dopamine transporter function. Proceedings of the National Academy of Sciences (U.S.A), 101: 372-377.

McDonald, S., Bennett, K. M. B., Chambers, H., \& Castiello, U. (1999) Covert orienting and focusing of attention in children with attention deficit hyperactivity disorder. Neuropsychologia, 37: 345-356.

Oades, R. D. (1985) The role of noradrenaline in tuning and dopamine in switching between signals in the CNS. Neuroscience and Biobehavioral Reviews, 9: 261-283.

Oades, R. D. (1997) Stimulus dimension shifts in patients with schizophrenia, with and without paranoid hallucinatory symptoms, or obsessive compulsive disorder: strategies, blocking and monoamine status. Behavioural Brain Research, 88: 115132

Oades, R. D. (1999) Dopamine: Go/No-Go motivation versus switching. Commentary on "Neurobiology of the structure of personality: dopamine, facilitation of incentive motivation, and extraversion", RA Depue \& PF Collins, The Behavioral and Brain Sciences, 22: 532-533.

Oades, R. D. (2000) Differential measures of sustained attention in children with attention-deficit/ hyperactivity or tic disorders: relationship to monoamine metabolism. Psychiatry Research, 93: 165-178.

Oades, R. D. (2002) Dopamine may be 'hyper' with respect to noradrenaline metabolism, but 'hypo' with respect to serotonin metabolism in children with
ADHD. Behavioural Brain Research, 130:97-101.

Oades, R. D. Slusarek, M., Velling, S. \& Bondy, B. (2002) Serotonin platelettransporter measures in childhood attention-deficit/hyperactivity disorder (ADHD): clinical versus experimental measures of impulsivity. World Journal of Biological Psychiatry, 3: 96-100.

Oie, M., Rund, B. R. \& Sundet, K. (1998) Covert visual attention in patients with early-onset schizophrenia. Schizophrenia Research, 34:195-205.

Rubia, K., Noorloos, J., Smith, A., Gunning, B. \& Sergeant, J. A. (2003) Motor timing deficits in community and clinical boys with hyperactive behavior: the effect of methylphenidate on motor timing. Journal of abnormal Child Psychology, 31: 301-311.

Silberstein, R. B., Farrow, M., Levy, F., Pipingas, A., Hay, D. A. \& Jarman, F. C. (1998) Functional brain electrical activity mapping in boys with attentiondeficit/hyperactivity disorder. Archives of General Psychiatry, 55: 1105-1112.

Sonuga-Barke, E. J. S. (2003) The dual pathway model of AD/HD: an elaboration of neuro-developmental characteristics. Neuroscience and Biobehavioral Reviews, 27: 593-604.

Toplak, M. E., Rucklidge, J. J., John, S. C. F., \& Tannock, R. (2003) Time perception deficits in attentiondeficit/hyperactivity disorder and comorbid reading difficulties in child and adolescent samples. Journal of Child Psychology and Psychiatry, 44:888-903.

Ucles, P., Lorente, S., \& Rosa, F. (1996) Neurophysiological methods testing the psychoneural basis of attention deficit hyperactivity disorder. Child's Nervous System, 12: 215-217. 
Target article abstract:

\title{
A dynamic developmental theory of attention-deficit/hyperactivity disorder (ADHD) predominantly hyperactive/impulsive and combined subtypes
}

\author{
Terje Sagvolden $^{1}$, Heidi Aase ${ }^{2}$, Espen Borgå Johansen ${ }^{3}$, Vivienne Ann Russell,
}

Behavioral and Brain Sciences (2005), 28, 397-468 - DOI:10.1017/S0140525X05000075

1. Department of Physiology, University of Oslo, NO-0317 Oslo, Norway

terje.sagvolden@medisin.uio.no

2. Norwegian Centre for the Studies of Behavioural Problems and Innovative Practice, Ltd., University of Oslo, NO-0118 Oslo, Norway heidiaa@atferd.unirand.no

3. Department of Physiology, University of Oslo, NO-0317 Oslo, Norway e.b.johansen@medisin.uio.no

4. Department of Human Biology, University of Cape Town, ZA-7925 South Africa russell@curie.uct.ac.za

\begin{abstract}
:
Attention-deficit/hyperactivity disorder (ADHD) is currently defined as a cognitive/behavioral developmental disorder where all clinical criteria are behavioral. Inattentiveness, overactivity, and impulsiveness are presently regarded as the main clinical symptoms. The dynamic developmental behavioral theory is based on the hypothesis that altered dopaminergic function plays a pivotal role by failing to modulate non-dopaminergic (primarily glutamate and GABA) signal transmission appropriately. A hypo-functioning mesolimbic dopamine branch produces altered reinforcement of behavior and deficient extinction of previously reinforced behavior. This gives rise to delay aversion, development of hyperactivity in novel situations, impulsiveness, deficient sustained attention, increased behavioral variability, and failure to "inhibit" responses ("disinhibition").

A hypo-functioning mesocortical dopamine branch will cause attention response deficiencies (deficient orienting responses, impaired saccadic eye movements, and poorer attention responses toward a target) and poor behavioral planning (poor executive functions). A hypo-functioning nigrostriatal dopamine branch will cause impaired modulation of motor functions and deficient nondeclarative habit learning and memory. These impairments will give rise to apparent developmental delay, clumsiness, neurological "soft signs," and a "failure to inhibit" responses when quick reactions are required. Hypo-functioning dopamine branches represent the main individual predispositions in the present theory. The theory predicts that behavior and symptoms in ADHD result from the interplay between individual predispositions and the surroundings. The exact ADHD symptoms at a particular time in life will vary and be influenced by factors having positive or negative effects on symptom development. Altered or deficient learning and motor functions will produce special needs for optimal parenting and societal styles. Medication will to some degree normalize the underlying dopamine dysfunction and reduce the special needs of these children. The theory describes how individual predispositions interact with these conditions to produce behavioral, emotional, and cognitive effects that can turn into relatively stable behavioral patterns.
\end{abstract}

\section{Keywords:}

catecholamine; clumsiness; dopamine; hyperkinesis; hyperkinetic disorder; impulsivity; monoamine; neuromodulator; overactivity; pollutants; reinforcement; reward; verbally governed behavior; soft signs; variability 